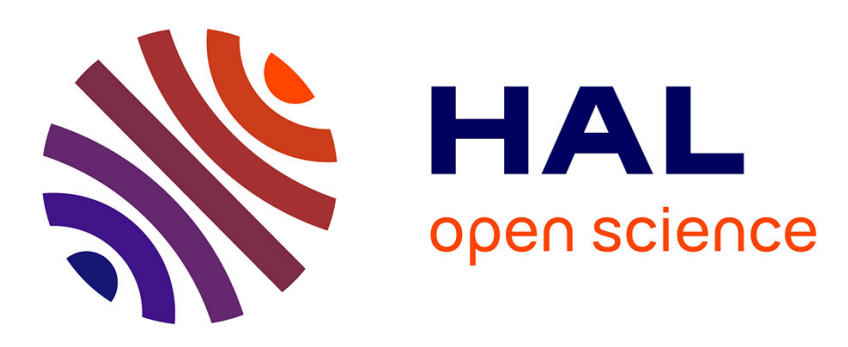

\title{
Temporal resolution enhancement of dynamic MRI sequences within a motion-based framework
}

\author{
K. Makki, B Borotikar, M Garetier, S Brochard, D Ben Salem, François
}

Rousseau

\section{- To cite this version:}

K. Makki, B Borotikar, M Garetier, S Brochard, D Ben Salem, et al.. Temporal resolution enhancement of dynamic MRI sequences within a motion-based framework. EMBC 2019: 41st Annual International Conference of the IEEE Engineering in Medicine and Biology Society, Jul 2019, Berlin, Germany. 10.1109/EMBC.2019.8857749 . hal-02286001

\section{HAL Id: hal-02286001 https://imt-atlantique.hal.science/hal-02286001}

Submitted on 13 Sep 2019

HAL is a multi-disciplinary open access archive for the deposit and dissemination of scientific research documents, whether they are published or not. The documents may come from teaching and research institutions in France or abroad, or from public or private research centers.
L'archive ouverte pluridisciplinaire HAL, est destinée au dépôt et à la diffusion de documents scientifiques de niveau recherche, publiés ou non, émanant des établissements d'enseignement et de recherche français ou étrangers, des laboratoires publics ou privés. 


\title{
Temporal resolution enhancement of dynamic MRI sequences within a motion-based framework
}

\author{
K. Makki ${ }^{1, \star}$, B. Borotikar ${ }^{2}$, M. Garetier ${ }^{3}$, S. Brochard ${ }^{2}$, D. Ben Salem ${ }^{2}$, F. Rousseau ${ }^{1}$
}

\begin{abstract}
Dynamic MRI has made it possible to noninvasively capture the moving human joints in vivo. Realtime Fast Field Echo (FFE) sequences have the potential to reduce the effect of motion artifacts by acquiring the image data within a few milliseconds. However, the short acquisition times affect the temporal resolution of the acquired sequences. In this paper, we propose a post-processing technique to reconstruct the missing frames of the sequence given the reduced amount of acquired data, which leads to recover the entire joint trajectory outside the MR scanner. To do this, we generalize the Log-Euclidean polyrigid registration framework to deal with dynamic three-dimensional articulated structures by adding the time as fourth dimension : we first estimate the rigid motion of each bone from the acquired data using linear intensity-based registration. Then, we fuse these local transformations to compute the non-linear joint deformations between successive images using a spatio-temporal log-euclidean polyrigid framework. The idea is to reconstruct the missing time frames by interpolating the realistic joint deformation fields in the domain of matrix logarithms assuming the motion to be consistent over a short period of time. The algorithm has been applied and validated using dynamic data from five children performing passive ankle dorsi-plantar flexion.
\end{abstract}

Index Terms-Dynamic MRI, tracking, intensity-based registration, diffeomorphisms, matrix analysis, eigenvalue

\section{INTRODUCTION}

Dynamic MRI was originally developed for cardiovascular imaging to quantify blood flow to study heart valve functions towards the end of the 1980s [1]. Since then, MRI acquisition and reconstruction techniques have improved by leaps and bounds with current sequences like fast-PC MRI, ultrafast MRI, and real-time FFE. These techniques have been successfully employed to quantify in vivo musculoskeletal biomechanics such as the ankle kinematics during dorsiplantar flexion. Sequences such as Cine-PC MRI or Fast-PC MRI [2] can provide velocity changes in the three directions of the space. However, these sequences require repeatable motions of the joint for up to two minutes (e.g. motion rate $=30$ cycles/minute for one dorsi plantarflexion cycle) and the dynamic data are built using these repeatable motions. Hence, velocity data does not meet the requirements of pediatric studies. Ultrafast Contrast-Enhanced MRI is another dynamic imaging technique which allows faster scanning speed by exploiting spatio-temporal redundancy and can acquire images during a single dorsi-plantar flexion cycle

\footnotetext{
* This work was supported by Région Bretagne, Chaire d'excellence INSERM-IMT Atlantique, Fondation de l'Avenir, and Fondation Motrice. Code: https://github.com/rousseau/dynMRI

${ }^{1}$ IMT Atlantique, LaTIM U1101 INSERM, UBL, Brest, France

${ }^{2}$ UBO, LaTIM U1101 INSERM, CHRU Brest, Brest, France

${ }^{3}$ HIA Brest, LaTIM U1101 INSERM, Brest, France
}

within 50 seconds [3]. However, these sequences require slow motion of the joint (mean rotation speed of $1^{\circ} / s$ ) as they are sensitive to motion artifacts so the motion itself is reduced to almost static or quasi-static nature where no real functionality can be assessed. Real-time FFE sequences [4] are less sensitive to motion artifacts and they can reduce the scanning time to only 18 seconds to acquire a single cycle of dorsi-plantar flexion. However, the short acquisition times reduce the number of acquired time frames. To resume, balancing the trade-off between acquisition speed and temporal resolution of dynamic sequences is still a challenging task. In this work, we propose to use FFE sequences, allowing the assessment of real functionalities (of muscles and tendons for example) with more comfortable and rapid acquisitions. The idea is to fastly acquire a reduced amount of data and then to estimate the missing amount with the help of image post-processing techniques. To this end, we propose to recover the missing data by continuously interpolating the temporal deformation field of the joint, which can be estimated using diffeomorphic registrations from the acquired data assuming the motion to be continuous and consistent during scanning. In this context, we have extended the Logeuclidean polyrigid framework proposed by Arsigny et al. [5] to deal with non-stationary dynamic MRI data by adding the time dimension into the stationary spatial framework. In the literature [6], [7], the exponential mapping for solving the polyrigid Ordinary Differential Equation (ODE) is recursively computed using the scaling and squaring method involving approximation theory for which the accuracy always remains debatable. In the work of Moler et al. [8], the authors have synthesized a study to compare the different existing techniques for calculating the matrix exponential (like methods involving approximation theory, differential equations, or matrix eigenvalues).Hence, we propose to use matrix eigendecomposition for exactly computing the exponential mapping since computations are performed in nondense regular grids due to the low-resolution of dynamic data, so that all the matrices can be stored in the main computer memory without the need of high-capacity RAM.

\section{DYNAMIC MRI SCANNING}

Five healthy children volunteers (between 10 and 14 years) participated in this pilot study. Each child was laid prone inside an Achieva dStream 3T MRI scanner (Philips Medical System, NL) with fully-extended knee, and two flex coils were placed on the ankle. The rate of ankle motion was standardized for all subjects using an MRI safe orthotic fixture (i.e. mean rotation speed of $4^{\circ} / \mathrm{s}$, each sequence captured 
the motion in 15 time frames). Real-time FFE sequences were used to acquire dynamic data during a single cycle of ankle plantal-dorsi flexion-extension (TR/TE=20.61/1.8, $\mathrm{FOV}=200 \times 200, \mathrm{FA}=15^{\circ}$, voxel $=0.57 \times 0.57 \times 8 \mathrm{~mm}$, acquisition time $=18 \mathrm{sec}$ ). For each child, two sequences during passive joint motion were acquired for repeatability measures.

\section{METHODS}

\section{A. Skeleton-tracking algorithm}

In this section, we present a robust skeleton-tracking algorithm employing intensity-based registration. For a given joint trajectory, each data-sequence is first splitted to $K$ 3D time frames ( $352 \times 352 \times 6$ voxels each one). Bones of interest (calcaneus, talus, and tibia) are manually segmented in the first or last time-frame and then idividually and automatically tracked throughout the sequence, forward or backward, using rigid registration. Our tracking method within the dynamic sequence is composed of two distinct steps :

1) Estimating bone rigid transforms by locally maximizing the correlation ratio between neighboring time frames using voxel-wise weighting based on the bone segmentations.

2) Propagating the bone segmentations forward or backward by applying the estimated rigid transforms using nearest neighbor interpolation.

Contrary to other interpolation methods like trilinear which induces smoothing of the bone mask, the use of nearest neighbor interpolation allows for preservation of bone shapes. To go forward in time, only one image interpolation is needed to propagate segmentations from the first time frame into each other. Since the composition of mappings in the Lie group $S E(3)$ corresponds to the multiplication of elementary transformations (i.e., $S E(3) \times \ldots \times S E(3)$ ), the direct path between images is computed by composing a set of estimated successive rigid transforms in order to avoid the propagation of interpolation errors as much as possible. The same for backward propagation for which the last time frame becomes the starting point for the tracking process. To improve the robustness of the algorithm, rotations are estimated based on their quaternion representations. Quaternions or versors are the most widely used in computer graphics and robotics, precisely in motion interpolation algorithms [9] as they offer a compact representation of rotations.

\section{B. Linear interpolation of bone transformation matrices}

Let $\mathrm{T}_{i, k}$ be the rigid transform from time frame $D_{k}$ to time frame $D_{k+1}$ for the bone $i$. Assuming that this transformation is diagonalizable (which is the case for most transformations in an orthonormal basis, such as the image coordinate system), then there exist an orthogonal matrix $P$ and a diagonal matrix $D$ such that $\mathrm{T}_{i, k}=P D P^{-1}$. The nonzero elements of $D$ are the eigenvalues of $\mathrm{T}_{i, k}\left(\lambda_{1}, \lambda_{2}, \lambda_{3}\right.$, and $\lambda_{4}=1$ ), while the columns of $P$ are the corresponding eigenvectors. Based on this matrix eigendecomposition, one can define $\mathrm{T}_{i, k}$ raised to its $\delta k^{t h}$ power as follows :

$$
\mathrm{T}_{i, k}^{\delta k}=P D^{\delta k} P^{-1}=P \cdot \operatorname{diag}\left(\lambda_{1}^{\delta k}, \lambda_{2}^{\delta k}, \lambda_{3}^{\delta k}, 1\right) . P^{-1}
$$

Integrating $\delta k$ continuously from 0 to 1 will infinitesimally change the matrix $\mathrm{T}_{i, k}^{\delta k}$ from the identity to the matrix $\mathrm{T}_{i, k}$. This allows for interpolating between two bone poses from a realistic transformation matrix, expressed in homogeneous coordinates (i.e., in the Special Euclidean group $S E(3)$ ).

\section{Spatio-temporal Log-euclidean polyrigid framework}

The Log-euclidean polyrigid framework provides an efficient way to synthesize joint deformation fields with nice properties like invertibility and smoothness, independently of the way local bone rigid transformations are first estimated. Based on the algebraic properties of this framework (the fact that it outputs diagonalziable velocity components in the Lie Algebra se(3)), it is then possible to add the time dimension to the stationary framework ( since $\log \left(T^{\delta k}\right)=\delta k \cdot \log (T)$ ). Given a point $x$ in the $k^{t h}$ time frame $D_{k}$, the target location of this point in the interpolated time frame $D_{k+\delta k}$ (located between $D_{k}$ and $D_{k+1}$ ) can be computed as follows :

$$
\Phi_{D_{k} \rightarrow D_{k+\delta k}}(x)=\exp \left(\delta k \sum_{i=1}^{N} \tilde{w}_{D_{k}}^{i}(x) \log \left(\mathrm{T}_{i, k}\right)\right) \cdot x
$$

where $\Phi_{\delta k=0}(x)=x ; \delta k \in[0,1]$ is the time-term added to the stationary polyrigid fusion formula; $\mathrm{T}_{i, k}$ is an element of the Lie group $S E(3) ; N$ is the total number of rigid components; $\Phi_{D_{k} \rightarrow D_{k+\delta k}}$ is the infinitesimal deformation field from $D_{k}$ to the time frame to be interpolated $D_{k+\delta k} ; \tilde{w}_{D_{k}}^{i}$ is a normalized weighting function (i.e., $\sum_{i=1}^{N} \tilde{w}_{D_{k}}^{i}(x)=$ $1, \forall x \in D_{k}$ ) which defines the local influence of the bone $i$ on the final voxel transformation (see section III-D).

In the literature, the exponential map of (2) is approximated using the scaling and squaring method [10]. In this work, this exponential map is computed exactly using eigendecomposition. Similarly to the matrix power defined in (1), the matrix exponential is obtained by exponentiating the associated eigenvalues (i.e., the non-zero elements of the diagonal matrix $D$ ). Thus, we just needed to cast the exponentiation of the complex eigenvalues of the transformation matrices in regular grids leading to smooth dynamic deformation fields that preserve the topology of the bones. Finally, the floating image intensities are mapped to new coordinates in the target image space by spline interpolation.

\section{Definition of weighting functions}

In the context of articulated registration, the choice of weight functions is very important as the main goal is to accurately estimate the non-linear joint deformations without affecting bone shapes. It is important to note that the Gaussian weight functions are more suitable for polyaffine fusion than the polyrigid fusion, as they do not guarantee the rigidity and accuracy of the transformations in the bonny skeleton (by affecting sharp peaks particularly while smoothing). In [7], Commowick et al. proposed an inverse-distance weighting function that preserves bone shapes after registration (3). However, such weighting functions yield inaccurate deformation outside the segmented bones.

$$
w_{D_{k}}^{i}(x)=\frac{1}{1+\alpha \operatorname{dist}\left(x, B_{D_{k}}^{i}\right)^{\beta}}
$$


where $B_{D_{k}}^{i}$ is the binary mask of bone $i$ in time frame $D_{k}$, and $\operatorname{dist}\left(x, B_{D_{k}}^{i}\right)$ is the Euclidean distance between $x$ and $B_{D_{k}}^{i}$.

Experiments show that an increase in $\beta$ leads to an increase in the deformation accuracy for non-rigid structures. Thus, we propose new weighting functions suited for articulated registration. These new weight functions, which are inversely proportional to a rate of the distance exponential can be used within the log-euclidean framework in order to obtain more realistic deformations outside the segmented bones :

$$
w_{D_{k}}^{i}(x)=\frac{2}{1+\exp \left(\gamma \operatorname{dist}\left(x, B_{D_{k}}^{i}\right)\right)}
$$

Note that these new weight functions yield more accurate transformations especially in the case of non-large deformations, which is always the case between two successive frames. $\gamma \in[0.4,0.8]$ to ensure smooth interpolation.

\section{Results}

The proposed pipeline has been applied on five dynamic MRI sequences. The exponential map of equation (2) is computed within $3 \mathrm{~s}$ on an Intel ${ }^{\circledR}$ Xeon ${ }^{\circledR}$ Processor E3-1271 v3 $3.60 \mathrm{GHz}$ on a $352 \times 352 \times 6$ regular grid using the proposed eigendecomposition method. Our code is implemented in Python $^{T M}$ using the LAPACK (Linear Algebra Package) routines for computing eigenvalues and eigenvectors. The RMSE between Achilles tendon contours was around $2 \mathrm{~mm}$ when using the weigthing functions defined in in equation (3) with $\beta<3$. Thus, the new definition of weighting functions played a crucial role in the enhancement of deformation accuracy outside the segmented bones while protecting skeleton edges, as reported in table I. Figure 1 illustrates, for one subject, such high-temporal-resolution reconstructed data.

The robustness of the method and the accuracy of the results have been evaluated using a local leave-one-out crossvalidation technique. This was done by reconstructing each acquired time frame $D_{k}$ based on the deformation field halfway between $D_{k-1}$ and $D_{k+1}$ for $k \in\{2 \ldots K-1\}$. Meaning each image $D_{k}$ has been reconstructed from the image-intensities of $D_{k-1}$, where $T_{i, k}$ is the rigid transformation from time frame $D_{k-1}$ to time frame $D_{k+1}$ for the bone $i$ and $\delta k$ is set to 0.5 in (2) (in the case of forward tracking). This gave a set of reconstructed time frames $\left\{D_{k}^{\prime}\right\}_{k \in 2 \ldots K-1}$. Results on bone motion interpolation are reported in table I. The accuracy of estimated deformation fields was then validated by computing the DICE overlap between the manually delineated masks of bones of interest on each acquired time frame $D_{k}$ and the bone segmentations, automatically propagated onto each reconstructed time frame $D_{k}^{\prime}$. For all sequences, the DICE coefficient was greater than 0.82 indicating accurate propagations of bone masks over each dynamic sequence. In order to provide a meaningful information about the accuracy of estimated deformation fields outside the segmented bones, we have computed the RMSE (Root Mean Square Error) between contour-points of Achilles tendon on each acquired time frame $D_{k}$ and on each reconstructed time frame $D_{k}^{\prime}$. In all cases, the mean error was less than $1 \mathrm{~mm}$ (using the new weighting functions), indicating the accuracy of the estimated deformation fields for the non-rigid structures. Figure 2 illustrates the importance of the weighting functions when estimating and interpolating the temporal joint deformation field.

The proposed design for bone motion tracking is still robust in either direction (i.e., when going forward or backward in time). Note that this interpolation technique is still available in the case of polyaffine transformations, in where local transformations are affine (i.e. include additional degrees of freedom like the scaling and shearing parameters).

\section{CONClusion}

In this work, we have presented a motion-interpolationbased method for increasing the temporal resolution of anatomical dynamic MRI sequences. We have generalized the Log-Euclidean polyrigid registration framework to dynamic articulated structures and we have also proposed new weight functions which are well adapted to our context. The exponential map of the Log euclidean polyrigid framework is computed in an efficient and elegant way using matrix diagonalization-based techniques. To conclude, the proposed post processing technique aims to overcome the physical limitations related to real-time dynamic MR imaging algorithms which are generally based on compressed sensing theory [11], for which it is hard to fastly acquire the entire or nearly the entire joint trajectory inside the MR scanner because of the limited $k$-space sampling.

\section{REFERENCES}

[1] R. Pettigrew, "Dynamic cardiac MR imaging. Techniques and applications.," Radiologic Clinics of North America, vol. 27, no. 6, pp. 11831203, 1989.

[2] A. J. Behnam, D. A. Herzka, and F. T. Sheehan, "Assessing the accuracy and precision of musculoskeletal motion tracking using CinePC MRI on a 3.0 Tesla platform," Journal of biomechanics, vol. 44, no. 1 , p. $193,2011$.

[3] E. Clarke, J. Martin, A. d'Entremont, M. Pandy, D. Wilson, and R. Herbert, "A non-invasive, 3D, dynamic MRI method for measuring muscle moment arms in vivo : demonstration in the human ankle joint and achilles tendon," Medical Engineering \& Physics, vol. 37, no. 1, pp. 93-99, 2015.

[4] T. Schaeffter, S. Weiss, H. Eggers, and V. Rasche, "Projection reconstruction balanced FFE for interactive real-time cardiac imaging," Magnetic Resonance in Medicine : An Official Journal of the International Society for Magnetic Resonance in Medicine, vol. 46, no. 6, pp. 1238-1241, 2001.

[5] V. Arsigny, X. Pennec, and N. Ayache, "Polyrigid and polyaffine transformations : a novel geometrical tool to deal with non-rigid deformations-application to the registration of histological slices," Medical Image Analysis, vol. 9, no. 6, pp. 507-523, 2005.

[6] V. Arsigny, O. Commowick, N. Ayache, and X. Pennec, "A fast and log-euclidean polyaffine framework for locally linear registration," Journal of Mathematical Imaging and Vision, vol. 33, no. 2, pp. 222238, 2009.

[7] O. Commowick, V. Arsigny, A. Isambert, J. Costa, F. Dhermain, F. Bidault, P.-Y. Bondiau, N. Ayache, and G. Malandain, "An efficient locally affine framework for the smooth registration of anatomical structures," Medical Image Analysis, vol. 12, no. 4, pp. 427-441, 2008.

[8] C. Moler and C. Van Loan, "Nineteen dubious ways to compute the exponential of a matrix, twenty-five years later," SIAM review, vol. 45 , no. 1, pp. 3-49, 2003. 
TABLE I: 3D DICE scores (for bones) and RMSE (for Achilles tendon contours) between manual segmentations of each structure of interest from the acquired data and segmentations of the same structures automatically-propagated onto the reconstructed data. Results are presented for several time frames across all subjects.

\begin{tabular}{|c|c|c|c|c|c|c|c|}
\hline Time & $k=2$ & $k=4$ & $k=6$ & $k=8$ & $k=10$ & $k=12$ & $k=14$ \\
\hline Calcaneus & $0.88 \pm 0.3$ & $0.83 \pm 0.4$ & $0.84 \pm 0.3$ & $0.84 \pm 0.2$ & $0.88 \pm 0.5$ & $0.85 \pm 0.3$ & $0.82 \pm 0.1$ \\
\hline Talus & $0.84 \pm 0.2$ & $0.84 \pm 0.4$ & $0.83 \pm 0.2$ & $0.85 \pm 0.4$ & $0.83 \pm 0.3$ & $0.84 \pm 0.3$ & $0.82 \pm 0.2$ \\
\hline Tibia & $0.9 \pm 0.3$ & $0.85 \pm 0.2$ & $0.88 \pm 0.5$ & $0.84 \pm 0.3$ & $0.88 \pm 0.2$ & $0.91 \pm 0.3$ & $0.92 \pm 0.2$ \\
\hline A. tendon & $0.45 \pm 0.04 \mathrm{~mm}$ & $0.7 \pm 0.12 \mathrm{~mm}$ & $0.56 \pm 0.08 \mathrm{~mm}$ & $0.6 \pm 0.1 \mathrm{~mm}$ & $0.7 \pm 0.07 \mathrm{~mm}$ & $0.8 \pm 0.18 \mathrm{~mm}$ & $0.6 \pm 0.1 \mathrm{~mm}$ \\
\hline
\end{tabular}

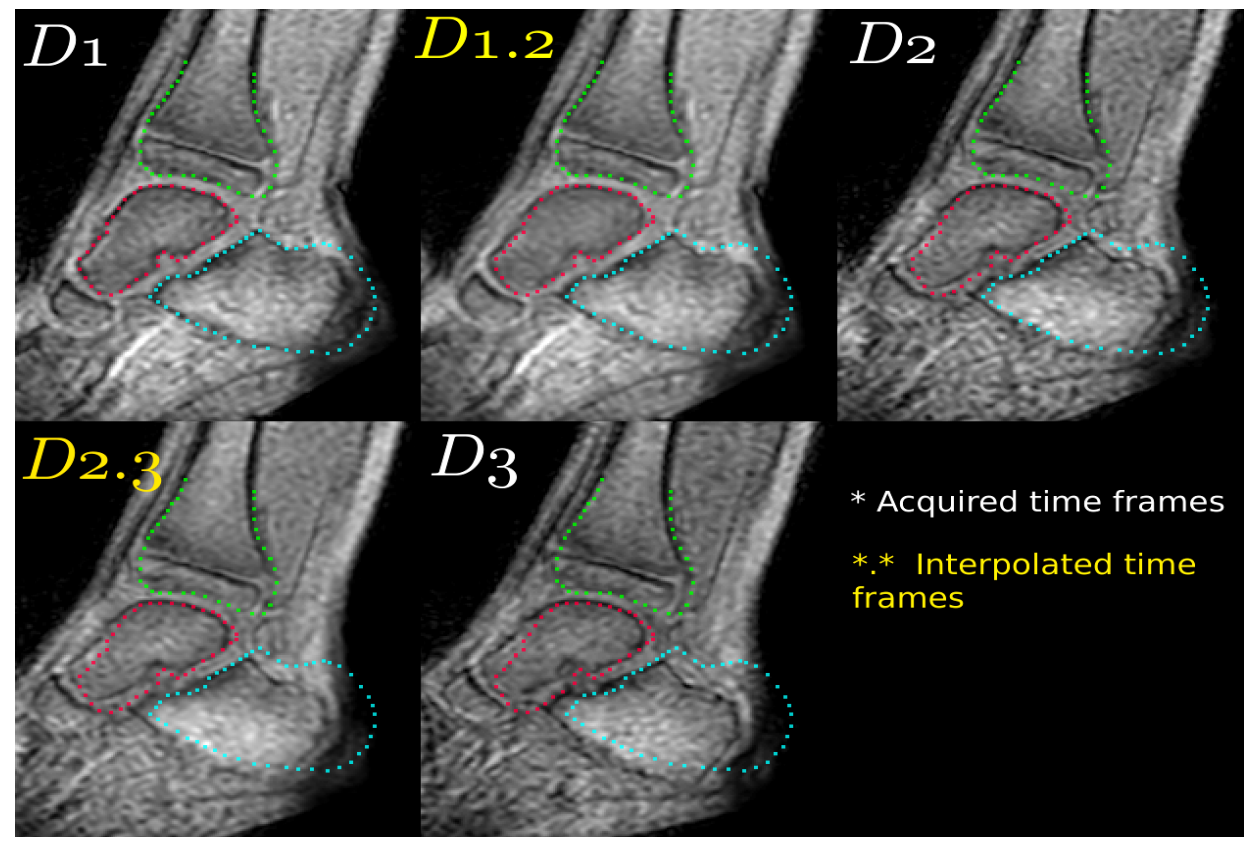

Fig. 1: Interpolation of missing time frames using the proposed forward tracking method $: D_{k}$ is the $k^{\text {th }}$ acquired time frame; while $D_{k . k+1}$ is the time frame half way between $D_{k}$ and $D_{k+1}$ (i.e., $\delta k=0.5$ in (2)). for $k=1 \ldots 3$.

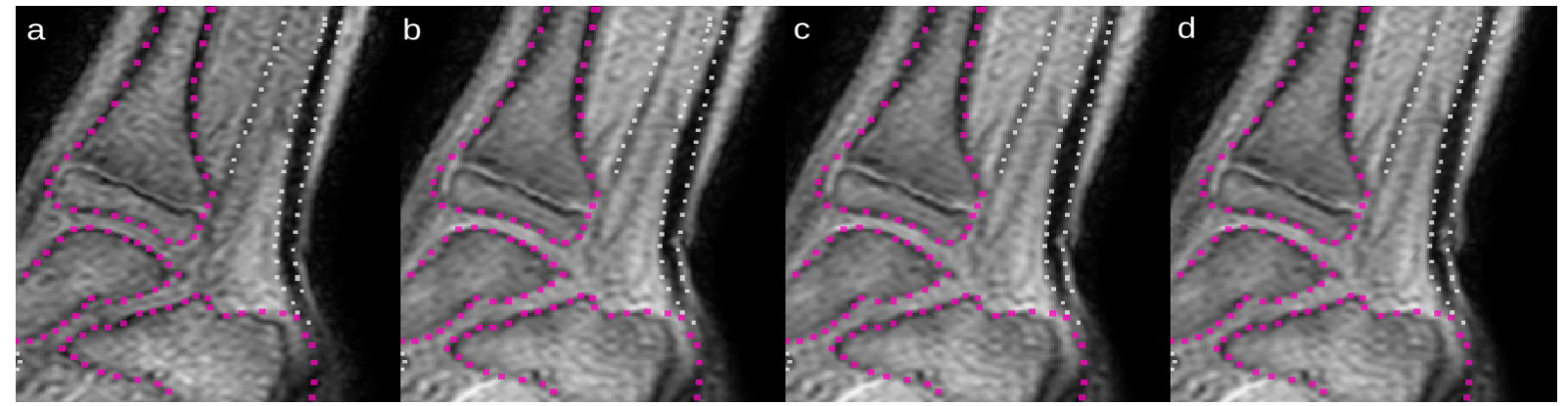

Fig. 2: Effects of different weighting functions on estimated deformation fields : (a) Target image; (b) Reconstructed image using the weighting functions defined in (3) with $\alpha=0.5$ and $\beta=1$; (c) Reconstructed image using the weighting functions defined in (3) with $\alpha=0.5$ and $\beta=2$; (d) Reconstructed image using our weighting functions defined in (4) with $\gamma=0.4$. The contours of bones (magenta) and of Achilles tendon (white) have been drawn in the target image to show the reconstruction accuracies when changing the weighting functions. Despite the fact that all the used weighting functions conserve bone topologies (this was validated by checking that the Jacobian map associated to the joint deformation field is equal to 1 over bone segmentations), the reconstructed image is more accurate for non-rigid structures such as the Achilles tendon especially when using our proposed weighting functions (d).

[9] R. Mukundan, "Quaternions : From classical mechanics to computer graphics, and beyond," in Proceedings of the 7th Asian Technology conference in Mathematics, pp. (2002) 97-105, 2002.

[10] N. J. Higham, "The scaling and squaring method for the matrix exponential revisited," SIAM Journal on Matrix Analysis and Applications, vol. 26, no. 4, pp. 1179-1193, 2005

[11] U. Gamper, P. Boesiger, and S. Kozerke, "Compressed sensing in dynamic MRI," Magnetic Resonance in Medicine : An Official Journal of the International Society for Magnetic Resonance in Medicine, vol. 59, no. 2, pp. 365-373, 2008. 